\title{
The best options in superior capsular reconstruction
}

\author{
Dong Hyun Kim, Young Soo Jung, Kyung-Rock Kim, Jong Pil Yoon \\ Department of Orthopedic Surgery, School of Medicine, Kyungpook National University, Daegu, Korea
}

\begin{abstract}
Irreparable massive rotator cuff tears cause pain, loss of function, and a decrease in range of motion, which cause serious disturbances in daily life. Young patients, in particular, are active and have relatively high functional requirements, and their surgical options are limited. Superior capsular reconstruction (SCR) was first proposed for irreparable massive rotator cuff tears, good clinical results have been reported in short-term follow up. Since then, SCR has been used increasingly worldwide for irreparable massive rotator cuff tears, and various studies have been published on clinical outcomes, biomechanical outcomes, surgical techniques, and graft types. This article reviews the optimal graft and surgical options for improving clinical outcomes in SCR.
\end{abstract}

Keywords: Rotator cuff; Shoulder; Superior capsular reconstruction; Tendon

\section{INTRODUCTION}

Irreparable massive rotator cuff tears cause pain, loss of function, and a decrease in range of motion, which cause serious disturbances in daily life $[1,2]$. In addition, since repairing irreparable massive rotator cuff tears have difficulty in complete footprint coverage and a high frequency of re-rupture, the postoperative clinical results often are unsatisfactory $[3,4]$. For this reason, in elderly patients with relatively few functional requirements, there are various surgical options for massive rotator cuff tears, such as debridement, biceps tenotomy/tenodesis, partial repair, tendon transfer, and reverse total shoulder arthroplasty (RTSA) [5-7]. However, since young patients are active and have relatively high functional requirements, these surgical options are limited to tendon transfer and patch augmentation [8,9]. In addition, although previous studies have reported that RTSA has good clini- cal result in elderly patients with massive rotator cuff tears, RTSA in active young patients under the age of 65 demonstrates relatively high complications and failure rates, and it is not an optimal surgical option in terms of longevity [10-12].

Mihata et al. [13,14] first proposed superior capsular reconstruction (SCR) using autologous tissue for irreparable massive rotator cuff tears, and they reported good clinical results in shortterm follow up. Since then, SCR has been increasingly used for irreparable massive rotator cuff tears, and various studies have been published on clinical outcomes, biomechanical outcomes, surgical techniques, and graft types. A previous systematic review reported that SCR reduced acromial contact pressure, produced superior humeral translation, and improved short-term clinical outcomes $[15,16]$. However, biologic healing at the contact surface between graft and bone is the most important factor to improve mid- and long-term outcomes of SCR [16,17]. Therefore,

Received: March 17, $2021 \quad$ Revised: May 6, 2021 Accepted: May 6, 2021

Correspondence to: Jong Pil Yoon

Department of Orthopedic Surgery, Kyungpook National University Hospital, 130 Dongdeok-ro, Jung-gu, Daegu 41944, Korea

Tel: +82-53-420-5628, Fax: +82-53-422-6605, E mail: jpyoon@knu.ac.kr, ORCID: https://orcid.org/0000-0001-6446-6254

Financial support: This study was supported in part by the Basic Science Research Program through the National Research Foundation of Korea funded by the Ministry of Science, ICT, and Future Planning. (NRF-2019R1A2C1003618).

Conflict of interest: None. 
for optimal biologic healing, selection of an optimal graft and appropriate surgical technique must be considered. For this reason, this article reviews the optimal graft and surgical options for improving clinical outcomes in SCR.

\section{GRAFT OPTIONS}

Mihata et al. [13] originally used a fascia lata autograft to reconstruct the superior capsule. Although their early results are promising, the fascia lata graft is thin, requiring doubling of the construct and a relatively large donor incision for harvesting. As an alternative, acellular dermal allografts were proposed for their theoretical advantages of reduced donor site morbidity, shorter associated operative times, ease of preparation, and strength of the graft $[18,19]$. Most of the previously reported studies described SCR using fascia lata graft or dermal allograft (Table 1) [20-29], Various biomechanical studies and clinical results report their use in SCR. Recently, however, various other autografts, allografts, xenografts, and synthetic grafts have been used in SCR, and the clinical results have been reported (Table 2) [30-34].

\section{Tensor Fascia vs. Dermal Allograft}

In a biomechanical cadaveric study comparing a fascia lata graft and a human dermal graft, single-layered human dermal allografts partially restored superior glenohumeral stability, whereas a fascia lata allograft completely restored superior glenohumeral stability [35]. A biomechanical comparison on the effect of SCR using a 3- and 6-mm-thick acellular dermal allograft demonstrated that SCR with a 6-mm-thick acellular dermal allograft better restored normal glenohumeral joint position and forces compared with a 3-mm-thick graft [36]. A biomechanical characterization of SCR using a fascia lata allograft, double layer dermal allograft, and single layer dermal allograft revealed that all three graft types can restore superior translation and subacromial contact pressure depending on the glenohumeral abduction angle, and fascia lata and double layer dermis can be more effective than single layer dermis [37]. SCR with a single 6-mm-thick acellular dermal allograft is non-inferior to fascia lata regarding subacromial space distance and peak subacromial contact pressures while restoring the superior stability of the glenohumeral joint compared to an intact joint [38].

Mihata et al. [39] reported the functional and radiographic results of SCR after 5 years of follow-up. Healed arthroscopic superior capsule reconstruction restored shoulder function and resulted in high rates of return to recreational sport and work. None of the 27 patients who experienced graft healing showed progression of cuff tear arthropathy, but all three patients with a graft tear had severe cuff tear arthropathy at 5 years postoperatively. A systematic review described two studies using only a fascia lata autograft and three studies using only a human dermal allograft, and the mean follow-up time ranged from 12 to 48 months. All studies reported statistically significant and clinically important mean improvements in active elevation (range of means, $28^{\circ}-56^{\circ}$ ), the Constant score (range of means, 12-47.1 points), or the American Shoulder and Elbow Surgeons (ASES) score (range of mean, 29.3-56 points). In total, 218 shoulders underwent postoperative magnetic resonance imaging. The graft tear rate reported in the studies using a fascia lata autograft (181 shoulders) ranged from $5 \%$ to $32 \%$, whereas the values reported in studies using a human dermal allograft (37 shoulders) ranged from $20 \%$ to $75 \%$ [40]. Another systematic review reported five fascia lata autograft clinical studies and four only-human dermal allograft clinical studies. Increases in ASES scores, forward elevation and external rotation values, and acromiohumeral distance (AHD) were found in all clinical fascia lata autograft studies. The human dermal allograft clinical studies reported increases in ASES scores, forward elevation values, and AHD but decreases in VAS scores [16].

\section{Other Graft Materials}

In SCR, there are not many studies on grafts other than fascia lata and dermal graft. However, as with patch augmentation in massive rotator cuff tears, various types of grafts are being tried. Few reports on the clinical outcome of these various grafts exist, and the surgical techniques are still mostly introduced.

\section{Biceps autograft}

The long head of the biceps tendon (LHBT) is a local autograft; the patient does not have donor site morbidity. All procedures are performed on the same shoulder, reducing operative time and risk of infection. Preserved vascular supply of the transposed LHBT can improve healing progression of rotator cuff repair. There is no extra cost for an allograft or an artificial graft [31]. Few reports reveal the clinical outcome of SCR using LHBT, but it has been reported to have significant effects in several biomechanical studies $[41,42]$. Most reports describe the surgical technique of SCR using LHBT [31,43-46].

El-Shaa et al. [42] reported in a study of 10 cadaveric shoulders that SCR with an LHBT autograft is a feasible procedure that is biomechanically equivalent and potentially even stronger than SCR with a fascia lata autograft for prevention of superior humeral migration. SCR with an LHBT autograft required $393.2 \% \pm 87.9 \%(p=0.029)$ of the force needed for superior humeral migration in massive rotator cuff tear, while SCR with a 


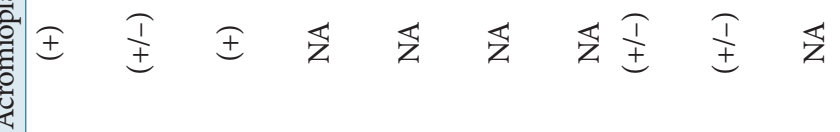

节

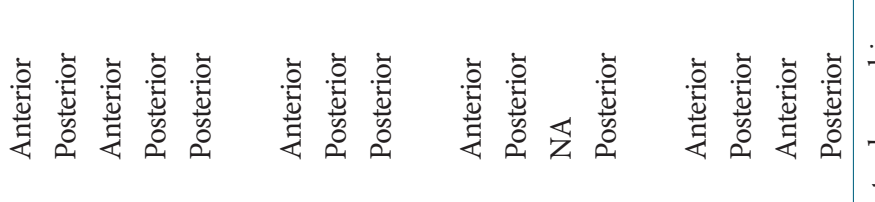

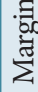

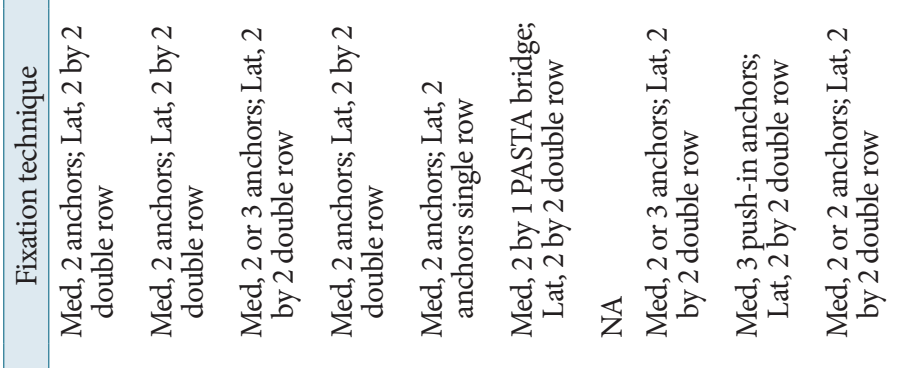

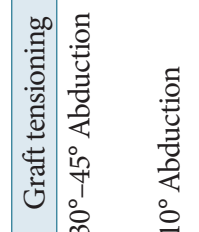

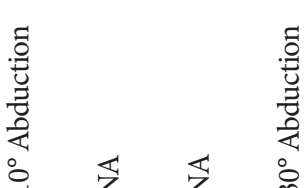

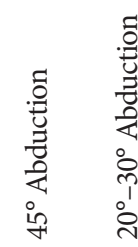

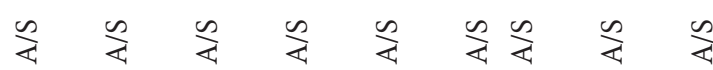

$\infty$
$i$
1

준

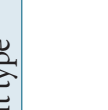

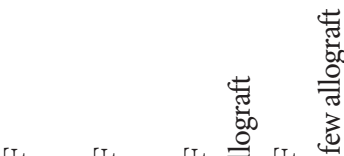

范

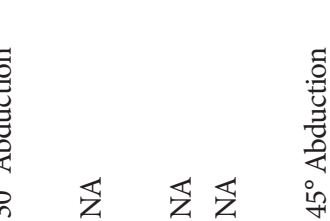

व:

政


Table 2. Graft options reported in SCR

\begin{tabular}{|c|c|}
\hline Category & Graft type \\
\hline Autografts & Fascia lata, biceps tendon, semitendinosus tendon \\
\hline Allografts & $\begin{array}{l}\text { Human dermal matrix, fascia lata, Achilles tendon, } \\
\text { semitendinosus tendon }\end{array}$ \\
\hline Synthetic grafts & Teflon Patch \\
\hline Xenografts & $\begin{array}{l}\text { Acellular porcine dermal xenograft, DX reinforce- } \\
\text { ment matrix }\end{array}$ \\
\hline
\end{tabular}

SCR: superior capsular reconstruction.

fascia lata autograft required $194.0 \% \pm 21.8 \%(\mathrm{p}=0.013)$. The LHBT reconstruction group trended toward a stronger reconstruction when normalized to the torn condition ( $p=0.059)$. Han et al. [41] reported a cadaveric study in seven shoulders. A modified SCR using LHBT both with and without side-to-side repair shifted the humeral head inferiorly at $30^{\circ}$ and $60^{\circ}$ of glenohumeral abduction, with the contact area further reduced at $60^{\circ}$. The techniques had comparable results for contact pressure and total rotational range of motion. They suggested that the LHBT with appropriate distal insertion on the greater tuberosity restores shoulder stability in irreparable rotator cuff tears by re-centering the humeral head on the glenoid.

Barth et al. [30] reported the clinical outcomes in 24 patients who underwent SCR with LHBT autograft. Twenty-four months after surgery, the group undergoing SCR with the LHBT showed improvement in absolute Constant score from 50 to 77, ASES from 45 to 80 , Subjective Shoulder Score from $41 \%$ to $75 \%$, the Simple Shoulder Test from 3.6 to 8.4 , and the visual analog scale (VAS) from 5.2 to 1.4. In all cases, the p-value was $<0.001$. The strength of the operated arm improved from $2.3 \mathrm{~kg}$ to $6.4 \mathrm{~kg}$ $(\mathrm{p}<0.001)$, and $91.7 \%(22 / 24)$ of the SCR group remained healed on ultrasound.

\section{Semitendinosus tendon autograft}

Rosales-Varo et al. [47] proposed an open technique for SCR with an autologous semitendinosus tendon graft in a reverse $\mathrm{V}$-shaped configuration (single fixation point on the glenoid and double fixation on the greater tuberosity). They found an improvement in Constant score from 49 (before SCR) to 77.25 (1 year after the operation). The mean active flexion significantly increased from $99.3^{\circ}$ to $142.5^{\circ}(\mathrm{p}<0.01)$. The mean preoperative AHD increased from 5.25 (before surgery) to 8.18 (after SCR). There were no tears of the graft during follow-up [47]. The technique is safe and reliable and has some potential advantages related to cost and lesser morbidity than the fascia lata autograft. Moreover, the tendon length allows for different configurations (double strand, V shaped, reverse V shaped, or box shaped). Mi- lano et al. [48] also reported an all-arthroscopic technique for superior capsule reconstruction using a doubled semitendinosus tendon autograft in a box-shaped configuration. They only reported the surgical technique, however, and not the clinical outcome.

\section{Patellar tendon allograft}

Croom et al. [49] reported SCR using a patellar tendon allograft (PT-SCR) in a study of eight cadaveric shoulders. They compared rotational range of motion, superior translation, and subacromial contact pressure, which were measured in the following experimental conditions: intact rotator cuff, massive rotator cuff tear, and PT-SCR. Application of the PT-SCR resulted in a decrease of superior translation compared with massive rotator cuff tear $(\mathrm{p}<0.001)$. At $0^{\circ}$ abduction $/ 60^{\circ}$ external rotation and $0^{\circ}$ abduction $/ 90^{\circ}$ external rotation, massive rotator cuff tear showed significantly greater peak subacromial contact pressure compared with the intact state $(\mathrm{p}<0.006)$. At both of these positions, PTSCR was able to reduce peak pressure to lower than or no significant difference from the intact state. There was no significant change in graft thickness, length, or width after testing. PT-SCR was able to reduce superior translation of the humeral head and peak subacromial contact pressure without restricting range of motion. Furthermore, there was no significant graft deformation during testing. They suggested that PT-SCR in this validated cadaveric model demonstrates favorable biomechanical properties and is a viable source of graft material for SCR.

\section{Achilles tendon-bone allograft}

Kim and Nam [33] reported arthroscopic SCR by the mini-open modified keyhole technique using an Achilles tendon-bone allograft. Using a keyhole to reduce graft tear in the greater tuberosity, they focused on saving operative time and the surface, such that bone-to-bone healing was induced by the tendon-bone graft. In another study on anterior cruciate ligament reconstruction, healing and revascularization were induced by grafting the Achilles tendon-bone through bone tunneling. In this way, they confirmed that the Achilles tendon-bone graft had a good effect in SCR by creating a keyhole-shaped tunnel in the humeral head. An appropriate size and thickness can be achieved with the Achilles tendon through appropriate folding due to the largesized graft. Although there is a risk of additional fractures due to artificial hole formation, it can be difficult to rehabilitate because bone healing should occur within a particular time frame. However, they followed the same rehabilitation protocol as that used in the general SCR technique. 


\section{Xenograft}

Acellular porcine dermal xenograft

Polacek [50] evaluated the short-term clinical outcomes and complications related to arthroscopic SCR with an acellular porcine dermal xenograft for treatment of irreparable massive rotator cuff tears in a total of 20 shoulders in 19 patients with rotator cuff tear. He reported mean range of motion, and the Shoulder Pain and Disability Index score showed significant improvement from $51.3 \%$ to $10.4 \%$ at 1-year follow-up. Active abduction improved from $65.4^{\circ}$ to $149.3^{\circ}$, and active forward flexion improved from $68.6^{\circ}$ to $151.4^{\circ}$ at 1-year follow-up. However, the procedure had a $30 \%$ complication rate, including a $15 \%$ rate of immunologic rejection of the xenograft [50].

\section{DX reinforcement matrix}

Kalina et al. [32] reported the clinical outcome of SCR using the DX Reinforcement (Arthrex, Naples, FL, USA) at 1 year (range, 6-18 months) in a total of 20 SCRs. The University of CaliforniaLos Angeles score improved from 10 to 29, the ASES from 23.8 to 73.2 , and the VAS from 7 to 2 . The mean active shoulder flexion was $74^{\circ}$ preoperatively and $161^{\circ}$ postoperatively. The mean active abduction was $74^{\circ}$ preoperatively and $161^{\circ}$ postoperatively. The mean active external rotation of the shoulder joint was $20^{\circ}$ preoperatively and $56^{\circ}$ postoperatively. The mean active external rotation at $90^{\circ}$ abduction was $21^{\circ}$ preoperatively and $82^{\circ}$ postoperatively. No complication specifically associated with use of xenograft has been reported.

\section{Teflon patch}

Teflon felt has been used for conventional patch graft surgery [51-53] (tendon reconstruction for rotator cuff tears), as well as in thoracic and cardiovascular surgery [54]. Okamura et al. [34] investigated the clinical and radiographic outcomes and postoperative complications of SCR using a Teflon graft of either 1 or 3 layers. They reported that SCR using a Teflon graft of either 1 or 3 layers significantly improved the ASES and VAS scores and muscle strength in shoulder abduction, with low rates of graft tears and complications after surgery.

\section{GRAFT THICKNESS AND SIZE}

In a previous cadaveric study, the thickness of the superior capsule of the normal glenohumeral joint was reported from $4.1 \mathrm{~mm}$ to $9.1 \mathrm{~mm}$ [55]. Biomechanically, although both $4-\mathrm{mm}$ - and 8-mm-thick fascia lata allografts reduced subacromial peak pressure after SCR, only the thicker 8-mm graft was able to decrease superior translation [56]. In an acellular dermal allograft, using a 6-mm-thick graft demonstrated similar maximum abduction angle, glenohumeral superior translation, subacromial peak contact pressure, and cumulative deltoid force compared with the intact state. When comparing the 3-mm- to the 6-mm-thick graft, significant differences were found in glenohumeral superior translation [36]. When comparing the 6-mm-thick acellular dermal allograft and the 8-mm-thick fascia lata allograft, SCR with a single, 6-mm-thick, acellular dermal allograft is non-inferior to a fascia lata tendon regarding subacromial space distance and peak subacromial contact pressures while restoring the superior stability of the glenohumeral joint compared to the intact state [38]. These results reveal that graft thickness determines the degrees of superior migration through compression of the humeral head. So, to visualize this effect adequately, graft thickness should be at least $6 \mathrm{~mm}$ in SCR.

\section{GRAFT TENSIONING (FIXATION AN- GLE)}

For optimal fixation, graft tensioning has been implicated to play a major role in glenohumeral contact properties and forces [56]. In addition, previous literature suggests differences in ideal abduction angles during SCR graft fixation based on type of material used intraoperatively $[20,56]$. One method for tensioning the graft is through abduction of the glenohumeral joint during fixation. Mihata et al. [56] suggested that $8 \mathrm{~mm}$ graft thickness and an arm position between $15^{\circ}$ and $45^{\circ}$ of shoulder abduction are the major aspects for sufficient reconstruction of the superior capsule. Various abduction angles using dermal allografts have been described, ranging from $20^{\circ}$ to $45^{\circ}$ of clinical shoulder abduction $[18,57]$. However, the optimum graft tension for best results in SCR remains unclear. Adams et al. [58] reported that SCR with anterior and posterior margin convergence tensioned at $15^{\circ}$ of glenohumeral abduction showed similar deltoid abduction force requirements to the native state in their biomechanical study. Dyrna et al. [59] reported in a biomechanical study of 10 fresh-frozen cadaveric shoulders that SCR using a graft fixed under tension (30 to $35 \mathrm{~N}$ ) demonstrated a significant increase in maximum shoulder abduction compared with a nontensioned graft $\left(65.0^{\circ} \pm 12.6^{\circ}\right.$ vs. $\left.54.1^{\circ} \pm 16.1^{\circ}, \mathrm{p}=0.04\right)$. However, abduction remained significantly less than in the intact state $\left(79.8^{\circ} \pm 5.8^{\circ}, \mathrm{p}=0.04\right)$. A tensioned SCR restored a maximum abduction of $81 \%$ of the native condition. Clinically, most surgeons have performed SCR graft fixation at $10^{\circ}$ to $45^{\circ}$ shoulder abduction per Mihata's recommendation [21-24]. But, there is a lack of clear clinical evidence for optimal abduction angle based on graft type in SCR. 


\section{MARGIN CONVERGENCE SUTURING (SIDE-TO-SIDE SUTURING)}

Mihata et al. [13] recommended side-to-side suturing between the graft and infraspinatus tendon or subscapularis tendon when performing SCR in irreparable rotator cuff tears. In biomechanical studies, SCR without side-to-side suturing did not decrease glenohumeral superior translation, although subacromial peak contact pressure decreased [60]. Adding side-to-side suturing restored superior stability to the intact level by achieving capsular continuity in the transverse direction. Total ROM did not decrease significantly after SCR with or without side-to-side suturing when the graft size matched the defect size [60].

\section{ACROMIOPLASTY}

Although SCR can increase the AHD, friction can be induced on the undersurface of the graft and acromion through movement after surgery, and this can cause a tear in the graft. Mihata et al. [13] recommended acromioplasty during SCR for irreparable rotator cuff tears. They also investigated the effects of acromioplasty on shoulder biomechanics after superior capsule reconstruction for irreparable supraspinatus tendon tears [61]. Including acromioplasty decreased the subacromial contact area without increasing the subacromial contact pressure in SCR. Clinically, it is not necessary to perform acromioplasty in SCR, and acromioplasty was not necessarily performed in other studies. However, most surgeons performed acromioplasty if there was evidence of abrasion on the undersurface of the acromion [24,62,63].

\section{CONCLUSION}

Since Mihata et al. [13] introduced SCR for irreparable rotator cuff tear several years ago, various studies on SCR have been conducted, and considerable progress has been made. The longterm clinical results of SCR are insufficient, however, and further studies on the various factors necessary for the best results in irreparable rotator cuff tear are needed. Knowledge of these various factors that must be considered for a successful SCR will lead to a better life for patients with massive rotator cuff tears.

\section{ORCID}

\author{
Dong Hyun Kim \\ Young Soo Jung \\ Kyung-Rock Kim \\ Jong Pil Yoon
}

https://orcid.org/0000-0001-9078-5953

https://orcid.org/0000-0003-2518-2560

https://orcid.org/0000-0002-1203-2742

https://orcid.org/0000-0001-6446-6254

\section{REFERENCES}

1. Tokish JM, Alexander TC, Kissenberth MJ, Hawkins RJ. Pseudoparalysis: a systematic review of term definitions, treatment approaches, and outcomes of management techniques. J Shoulder Elbow Surg 2017;26:e177-87.

2. Werner CM, Steinmann PA, Gilbart M, Gerber C. Treatment of painful pseudoparesis due to irreparable rotator cuff dysfunction with the Delta III reverse-ball-and-socket total shoulder prosthesis. J Bone Joint Surg Am 2005;87:1476-86.

3. Bedi A, Dines J, Warren RF, Dines DM. Massive tears of the rotator cuff. J Bone Joint Surg Am 2010;92:1894-908.

4. Oh JH, Kim SH, Kang JY, Oh CH, Gong HS. Effect of age on functional and structural outcome after rotator cuff repair. Am J Sports Med 2010;38:672-8.

5. Thorsness R, Romeo A. Massive rotator cuff tears: trends in surgical management. Orthopedics 2016;39:145-51.

6. Colvin AC, Egorova N, Harrison AK, Moskowitz A, Flatow EL. National trends in rotator cuff repair. J Bone Joint Surg Am 2012;94:227-33.

7. Duquin TR, Buyea C, Bisson LJ. Which method of rotator cuff repair leads to the highest rate of structural healing?: a systematic review. Am J Sports Med 2010;38:835-41.

8. Flury M. Patch augmentation of the rotator cuff: a reasonable choice or a waste of money? Orthopade 2016;45:136-42.

9. Ciampi P, Scotti C, Nonis A, et al. The benefit of synthetic versus biological patch augmentation in the repair of posterosuperior massive rotator cuff tears: a 3-year follow-up study. Am J Sports Med 2014;42:1169-75.

10. Otto RJ, Clark RE, Frankle MA. Reverse shoulder arthroplasty in patients younger than 55 years: 2- to 12-year follow-up. J Shoulder Elbow Surg 2017;26:792-7.

11. Sershon RA, Van Thiel GS, Lin EC, et al. Clinical outcomes of reverse total shoulder arthroplasty in patients aged younger than 60 years. J Shoulder Elbow Surg 2014;23:395-400.

12. Ek ET, Neukom L, Catanzaro S, Gerber C. Reverse total shoulder arthroplasty for massive irreparable rotator cuff tears in patients younger than 65 years old: results after five to fifteen years. J Shoulder Elbow Surg 2013;22:1199-208.

13. Mihata T, Lee TQ, Watanabe C, et al. Clinical results of arthroscopic superior capsule reconstruction for irreparable rotator cuff tears. Arthroscopy 2013;29:459-70.

14. Mihata T, McGarry MH, Pirolo JM, Kinoshita M, Lee TQ. Superior capsule reconstruction to restore superior stability in irreparable rotator cuff tears: a biomechanical cadaveric study. Am J Sports Med 2012;40:2248-55.

15. Smith TJ, Gowd AK, Kunkel J, Kaplin L, Waterman BR. Superi- 
or capsular reconstruction provides sufficient biomechanical outcomes for massive, irreparable rotator cuff tears: a systematic review. Arthroscopy 2021;37:402-10.

16. Makovicka JL, Chung AS, Patel KA, Deckey DG, Hassebrock JD, Tokish JM. Superior capsule reconstruction for irreparable rotator cuff tears: a systematic review of biomechanical and clinical outcomes by graft type. J Shoulder Elbow Surg 2020;29: 392-401.

17. Zastrow RK, London DA, Parsons BO, Cagle PJ. Superior capsule reconstruction for irreparable rotator cuff tears: a systematic review. Arthroscopy 2019;35:2525-34.

18. Tokish JM, Beicker C. Superior capsule reconstruction technique using an acellular dermal allograft. Arthrosc Tech 2015; 4:e833-9.

19. Hirahara AM, Adams CR. Arthroscopic superior capsular reconstruction for treatment of massive irreparable rotator cuff tears. Arthrosc Tech 2015;4:e637-41.

20. Denard PJ, Brady PC, Adams CR, Tokish JM, Burkhart SS. Preliminary results of arthroscopic superior capsule reconstruction with dermal allograft. Arthroscopy 2018;34:93-9.

21. Burkhart SS, Pranckun JJ, Hartzler RU. Superior capsular reconstruction for the operatively irreparable rotator cuff tear: clinical outcomes are maintained 2 years after surgery. Arthroscopy 2020;36:373-80.

22. de Campos Azevedo CI, Ângelo AC, Vinga S. Arthroscopic superior capsular reconstruction with a minimally invasive harvested fascia lata autograft produces good clinical results. Orthop J Sports Med 2018;6:2325967118808242.

23. Lee SJ, Min YK. Can inadequate acromiohumeral distance improvement and poor posterior remnant tissue be the predictive factors of re-tear?: preliminary outcomes of arthroscopic superior capsular reconstruction. Knee Surg Sports Traumatol Arthrosc 2018;26:2205-13.

24. Pennington WT, Bartz BA, Pauli JM, Walker CE, Schmidt W. Arthroscopic superior capsular reconstruction with acellular dermal allograft for the treatment of massive irreparable rotator cuff tears: short-term clinical outcomes and the radiographic parameter of superior capsular distance. Arthroscopy 2018;34: 1764-73.

25. Mihata T, Lee TQ, Fukunishi K, et al. Return to sports and physical work after arthroscopic superior capsule reconstruction among patients with irreparable rotator cuff tears. Am J Sports Med 2018;46:1077-83.

26. Lim S, AlRamadhan H, Kwak JM, Hong H, Jeon IH. Graft tears after arthroscopic superior capsule reconstruction (ASCR): pattern of failure and its correlation with clinical outcome. Arch Orthop Trauma Surg 2019;139:231-9.
27. Yoon JY, Kim PS, Jo CH. Clinical and radiological results after arthroscopic superior capsular reconstruction in patients with massive irreparable rotator cuff tears. Clin Shoulder Elb 2018; 21:59-66.

28. Hirahara AM, Andersen WJ, Panero AJ. Superior capsular reconstruction: clinical outcomes after minimum 2-year follow-up. Am J Orthop (Belle Mead NJ) 2017;46:266-78.

29. Pogorzelski J, DelVecchio BM, Hussain ZB, Fritz EM, Godin JA, Millett PJ. Superior capsule reconstruction for massive rotator cuff tears: key considerations for rehabilitation. Int J Sports Phys Ther 2017;12:390-401.

30. Barth J, Olmos MI, Swan J, Barthelemy R, Delsol P, Boutsiadis A. Superior capsular reconstruction with the long head of the biceps autograft prevents infraspinatus retear in massive posterosuperior retracted rotator cuff tears. Am J Sports Med 2020;48: 1430-8.

31. Chiang CH, Shaw L, Chih WH, Yeh ML, Su WR. Arthroscopic rotator cuff repair combined with modified superior capsule reconstruction as reinforcement by the long head of the biceps. Arthrosc Tech 2019;8:e1223-31.

32. Kalina R, Neoral P, Holibka R, Gallo J. Arthroscopic superior capsule reconstruction using the $\mathrm{dx}$ reinforcement matrix in patients with irreparable rotator cuff tears: pilot data. Acta Chir Orthop Traumatol Cech 2019;86:264-70.

33. Kim JW, Nam DJ. Arthroscopic superior capsular reconstruction by the mini-open modified keyhole technique using an achilles tendon-bone allograft. Arthrosc Tech 2020;9:e275-81.

34. Okamura K, Abe M, Yamada Y, et al. Arthroscopic superior capsule reconstruction with Teflon felt synthetic graft for irreparable massive rotator cuff tears: clinical and radiographic results at minimum 2-year follow-up. J Shoulder Elbow Surg 2021;30:625-34.

35. Mihata T, Bui CN, Akeda M, et al. A biomechanical cadaveric study comparing superior capsule reconstruction using fascia lata allograft with human dermal allograft for irreparable rotator cuff tear. J Shoulder Elbow Surg 2017;26:2158-66.

36. Scheiderer B, Kia C, Obopilwe E, et al. Biomechanical effect of superior capsule reconstruction using a 3-mm and 6-mm thick acellular dermal allograft in a dynamic shoulder model. Arthroscopy 2020;36:355-64.

37. E Cline K, Tibone JE, Ihn H, et al. Superior capsule reconstruction using fascia lata allograft compared with double- and single-layer dermal allograft: a biomechanical study. Arthroscopy 2021;37:1117-25.

38. Shah SS, Kontaxis A, Jahandar A, et al. Superior capsule reconstruction using a single 6-mm-thick acellular dermal allograft for massive rotator cuff tears: a biomechanical cadaveric comparison 
to fascia lata allograft. J Shoulder Elbow Surg 2021;S10582746(20)30961-7.

39. Mihata T, Lee TQ, Hasegawa A, et al. Five-year follow-up of arthroscopic superior capsule reconstruction for irreparable rotator cuff tears. J Bone Joint Surg Am 2019;101:1921-30.

40. de Campos Azevedo CI, Andrade R, Leiria Pires Gago Ângelo AC, Espregueira-Mendes J, Ferreira N, Sevivas N. Fascia lata autograft versus human dermal allograft in arthroscopic superior capsular reconstruction for irreparable rotator cuff tears: a systematic review of clinical outcomes. Arthroscopy 2020; 36:579-91.

41. Han F, Kong CH, Hasan MY, Ramruttun AK, Kumar VP. Superior capsular reconstruction for irreparable supraspinatus tendon tears using the long head of biceps: a biomechanical study on cadavers. Orthop Traumatol Surg Res 2019;105:257-63.

42. El-Shaar R, Soin S, Nicandri G, Maloney M, Voloshin I. Superior capsular reconstruction with a long head of the biceps tendon autograft: a cadaveric study. Orthop J Sports Med 2018;6: 2325967118785365.

43. Kim YS, Lee HJ, Park I, Sung GY, Kim DJ, Kim JH. Arthroscopic in situ superior capsular reconstruction using the long head of the biceps tendon. Arthrosc Tech 2018;7:e97-103.

44. Kim D, Jang Y, Park J, On M. Arthroscopic superior capsular reconstruction with biceps autograft: snake technique. Arthrosc Tech 2019;8:e1085-92.

45. Adrian SC, Field LD. Biceps transposition for biological superior capsular reconstruction. Arthrosc Tech 2020;9:e841-6.

46. Fandridis E, Zampeli F. Superior capsular reconstruction with double bundle of long head biceps tendon autograft: the "box" technique. Arthrosc Tech 2020;9:e1747-57.

47. Rosales-Varo AP, Zafra M, García-Espona MA, Flores-Ruiz MA, Roda O. Superior capsular reconstruction of irreparable rotator cuff tear using autologous hamstring graft. Rev Esp Cir Ortop Traumatol 2019;63:1-6.

48. Milano G, Saccomanno MF, Colosio A, et al. Arthroscopic superior capsule reconstruction with doubled autologous semitendinosus tendon graft. Arthrosc Tech 2020;9:e1665-72.

49. Croom WP, Adamson GJ, Lin CC, et al. A biomechanical cadaveric study of patellar tendon allograft as an alternative graft material for superior capsule reconstruction. J Shoulder Elbow Surg 2019;28:1241-8.

50. Polacek M. Arthroscopic superior capsular reconstruction with acellular porcine dermal xenograft for the treatment of massive irreparable rotator cuff tears. Arthrosc Sports Med Rehabil 2019;1:e75-84.

51. Ozaki J, Fujimoto S, Masuhara K, Tamai S, Yoshimoto S. Reconstruction of chronic massive rotator cuff tears with synthetic materials. Clin Orthop Relat Res 1986;(202):173-83.

52. Seker V, Hackett L, Lam PH, Murrell GA. Evaluating the outcomes of rotator cuff repairs with polytetrafluoroethylene patches for massive and irreparable rotator cuff tears with a minimum 2-year follow-up. Am J Sports Med 2018;46:3155-64.

53. Sunwoo JY, Lam PH, Murrell GA. A comparison of two arthroscopic techniques for interpositional polytetrafluoroethylene patch repair for massive irreparable rotator cuff tears: speed and biomechanics. HSS J 2018;14:186-91.

54. Harrison JH. A teflon weave for replacing tissue defects. Surg Gynecol Obstet 1957;104:584-90.

55. Nimura A, Kato A, Yamaguchi K, et al. The superior capsule of the shoulder joint complements the insertion of the rotator cuff. J Shoulder Elbow Surg 2012;21:867-72.

56. Mihata T, McGarry MH, Kahn T, Goldberg I, Neo M, Lee TQ. Biomechanical effect of thickness and tension of fascia lata graft on glenohumeral stability for superior capsule reconstruction in irreparable supraspinatus tears. Arthroscopy 2016;32:418-26.

57. Adams CR, Denard PJ, Brady PC, Hartzler RU, Burkhart SS. The arthroscopic superior capsular reconstruction. Am J Orthop (Belle Mead NJ) 2016;45:320-4.

58. Adams CR, Comer B, Scheiderer B, et al. The effect of glenohumeral fixation angle on deltoid function during superior capsule reconstruction: a biomechanical investigation. Arthroscopy 2020;36:400-8.

59. Dyrna F, Berthold DP, Muench LN, et al. Graft tensioning in superior capsular reconstruction improves glenohumeral joint kinematics in massive irreparable rotator cuff tears: a biomechanical study of the influence of superior capsular reconstruction on dynamic shoulder abduction. Orthop J Sports Med 2020;8: 2325967120957424.

60. Mihata T, McGarry MH, Kahn T, Goldberg I, Neo M, Lee TQ. Biomechanical role of capsular continuity in superior capsule reconstruction for irreparable tears of the supraspinatus tendon. Am J Sports Med 2016;44:1423-30.

61. Mihata T, McGarry MH, Kahn T, Goldberg I, Neo M, Lee TQ. Biomechanical effects of acromioplasty on superior capsule reconstruction for irreparable supraspinatus tendon tears. Am J Sports Med 2016;44:191-7.

62. Badman BL, Baessler AM, Moor M. Short-term clinical outcomes and comparison of ultrasound versus magnetic resonance imaging of superior capsular reconstruction. Arthrosc Sports Med Rehabil 2020;2:e229-35.

63. Erratum to "A Biomechanical Cadaveric Study of Patella Tendon Allograft as an Alternative Graft Material for Superior Capsule Reconstruction" [J Shoulder Elbow Surg 2018;28:1241-1248]. J Shoulder Elbow Surg 2019;28:e357. 